\title{
Multiple Fault Location in a Photovoltaic Array Using Bidirectional Hetero-Associative Memory Network in Micro-Distribution Systems
}

\author{
Long-Yi Chang ${ }^{1, *}$, Neng-Sheng Pai ${ }^{1}$, Min-Hung Chou ${ }^{2}$, Jian-Liung Chen ${ }^{3}$, Chao-Lin Kuo ${ }^{4}$ \\ and Chia-Hung Lin ${ }^{1, *}$ \\ 1 Department of Electrical Engineering, National Chin-Yi University of Technology, Taiping District, \\ Taichung City 41170, Taiwan; pai@ncut.edu.tw \\ 2 Department of Marine Engineering, National Kaohsiung University of Science and Technology, Cijin District, \\ Kaohsiung City 80543, Taiwan; chouhp88scott@nkust.edu.tw \\ 3 Department of Electrical Engineering, Kao-Yuan University, Lu-Chu District, Kaohsiung City 82151, Taiwan; \\ t20047@cc.kyu.edu.tw \\ 4 Department of Maritime Information and Technology, National Kaohsiung University of Science and \\ Technology, Cijin District, Kaohsiung City 80543, Taiwan; clkuo@nkust.edu.tw \\ * Correspondence: lychang@mail.ncut.edu.tw (L.-Y.C.); eechl53@gmail.com (C.-H.L.)
}

Received: 24 May 2018; Accepted: 13 August 2018; Published: 15 August 2018

\begin{abstract}
In manual maintenance inspections of large-scaled photovoltaic (PV) or rooftop PV systems, several days are required to survey the entire PV field. To improve reliability and shorten the amount of time involved, this study proposes an electrical examination-based method for locating multiple faults in the PV array. The maximum power point tracking (MPPT) algorithm is used to estimate the maximum power of each PV panel; this is then compared with metering the output power of PV array. Power degradation indexes as input variables are parameterized to quantify the degradation between estimated maximum PV output power and metered PV output power, which can be categorized into normal condition, grounded faults, open-circuit faults, bridged faults, and mismatch faults. Bidirectional hetero-associative memory (BHAM) networks are then used to associate the inputs and locate multiple faults as output variables within the PV array. For a rooftop PV system with two strings, experimental results demonstrate that the proposed model has computational efficiency in learning and detection accuracies for real-time applications, and that its algorithm is easily implemented in a mobile intelligent vehicle.
\end{abstract}

Keywords: rooftop photovoltaic (PV) system; maximum power point tracking (MPPT); power degradation index; bidirectional hetero-associative memory network (BHAM)

\section{Introduction}

A photovoltaic array is a single electricity-producing unit in which several PV panel components are arranged in parallel (or in a series configuration) to increase output power. PV energy conversion systems use a solar tracking system with MPPT methods to improve output performance and battery charge solutions [1-3]. There are different sized PV systems, ranging from small, rooftop, or building-integrated systems with capacities $>10$ kilowatts to those with capacities of $>100$ 's of megawatts in concentrated solar, heating/cooling systems, and home power supplies. A PV system can operate in grid-connected and off-grid modes within a smart grid or within a small portion of the electricity market. In Taiwan, PV systems have niche usages in outdoor spaces due to the subtropical environment, and they are easy to install and operate noiselessly. However, the output power of a PV system is influenced by changes in environmental conditions, such as solar radiation and temperature. 
In addition, output power may be affected by any fault within the PV array, such as grounded faults, open circuit faults, and bridged faults [4-6]. Power fuses, overcurrent protection devices, and grounded fault protection devices can be employed to isolate grounded faults on DC-DC converter sites and DC-AC inverter sites, but as fault currents are also affected by environmental conditions, protection devices are unable to clear the fault under low solar radiation or during night-to-day transition. When there is a lower output current, MPPT methods can interfere to estimate the output current while a certain fault is identified, thereby triggering the current-based protection device [6,7]. It is difficult to locate faults via manual inspection within a PV array; therefore, an online assistant tool is necessary for use in locating multiple faults and conducting long-term outdoor monitoring. As output power is significantly altered by damage or faults occurring in one or more PV panels, this study proposes a bidirectional associative memory network to detect multiple faults within the PV array, as seen a schematic diagram in Figure 1.

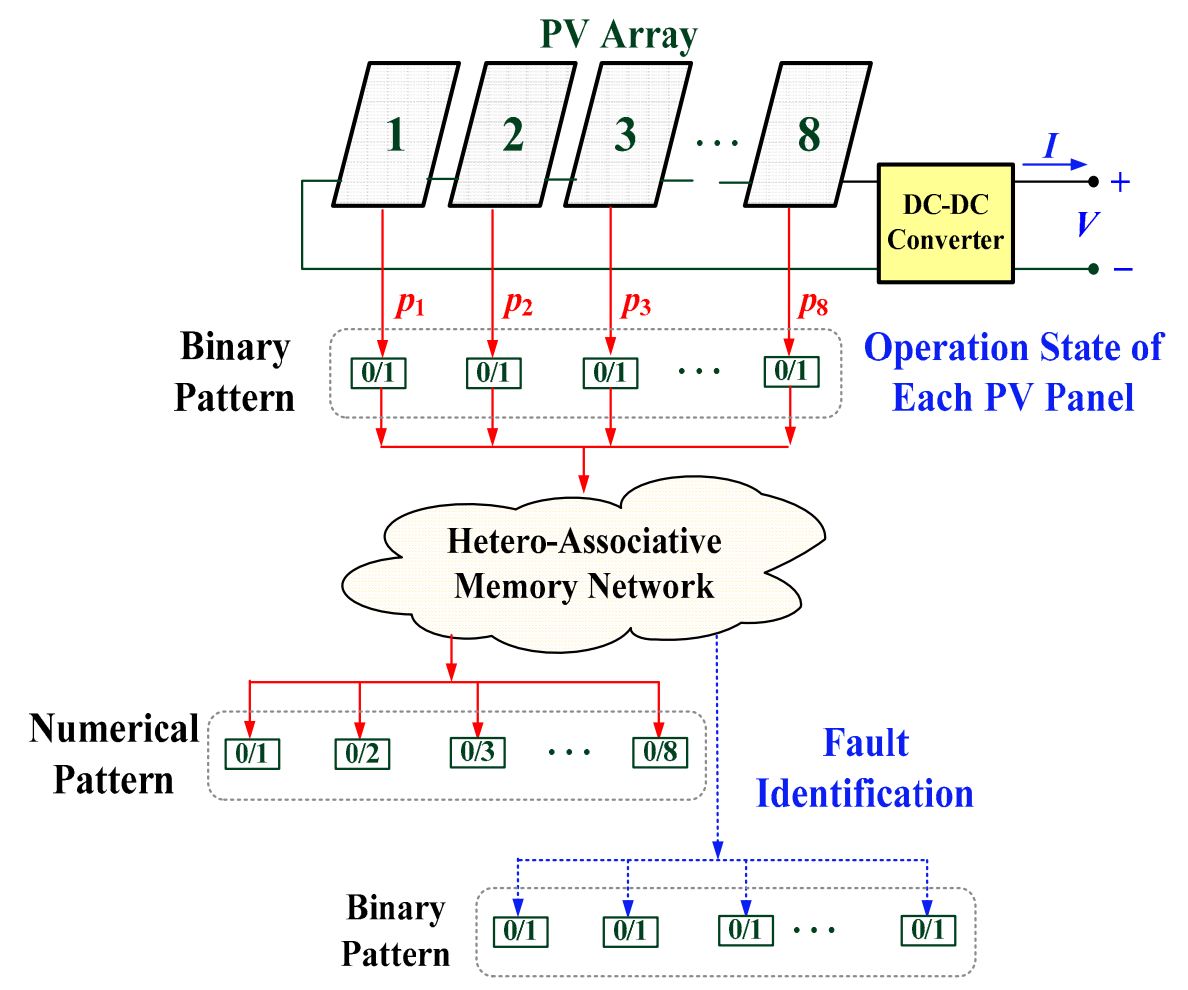

Figure 1. Schematic diagram of multiple fault location in a PV array using hetero-associative memory network.

Manual techniques are traditionally used to examine PV panels, such as individual inspections, imaging examinations, and electrical examinations. In the laboratory or during manufacture quality control processing, visual inspection, thermal imaging analysis, and infrared thermography (IRT) are commonly used to observe color changes and visible hot spots on thermal images or the IRT of solar panels. These methods are safe and involve no-contact measurements to check the solar panels for open circuits/short circuits in a cell string, cell ruptures, and cell or/and glass cracks [8-10]. However, such examinations are only used to protect both the customer and the contractor before solar panel installation, and require extra thermal imaging cameras or IRT to be mounted in front of the PV panel; thus, they are usually used in the laboratory to identify designs, materials, and cell flaws (IEC 61215/IEC 61646). To measure voltage and current on the DC-DC converter site or DC-AC inverter site, electrical examinations are needed; these are also required to determine and quantify output power degradation due to possible faulty panels in a PV array [6,11,12]. It is known that any fault in an array can cause output power degradation; thus conducting an electrical examination using 
current (I)-voltage $(\mathrm{V})$ or V-power $(\mathrm{P})$ curve analysis string by string is a reliable online testing method used to determine the locations of multiple faults.

On the DC-AC inverter side, the inverter has no overcurrent or overvoltage because a transformer provides galvanic isolation between the PV array and the utility grid. Power fuses, overcurrent, and ground-fault protection devices have been used to isolate ground faults in a conductor and any overcurrent from the PV array or fault on the grid-connected side. However, on the DC-DC converter side, the output power and currents can be degraded due to any fault within an array. Previous studies [6,7] have proposed that the use of the MPPT algorithm may prevent faults during periods of lower solar radiation and night-to-day transition, as the algorithm can locate the reference value at the maximum power point. When the measured output power is degraded, based on the electrical property, faults such as grounded faults, line-to-line faults, and open circuit faults can be identified [6,11,12]. Artificial intelligence (AI) methods, such as artificial neural network, support vector machine, and extreme learning machine, combined with the MPPT algorithm, can be employed to identify the fault classes under clear days, lower solar radiation conditions, and night-to-day transition. With sufficiently training data, a machine learning model with optimization algorithms can learn the correlations hidden in the training data via a nonlinear fitting process to perform the prediction and classification applications. These methods have utilized the machine learning model from a large experimental database to perform the prediction and optimization on a solar water system, such as the predictions of heat collection rates and heat loss coefficients to a water-in-glass evacuated tube solar water heater $[13,14]$. The AI methods are the well-known mathematic model for nonlinear curve fitting, numerical prediction, and classification applications. In this study, we will select the well-developed machine-learning model to design a fault location method in a PV array. However, AI-based classifiers need to determine the number of training patterns, number of hidden layers, number of hidden nodes, and network structure, and to adjust optimal parameters using the optimization algorithms [13,14].

Based on an electrical examination, this study proposes the use of the bidirectional hetero-associative memory (BHAM) network [15-18] to detect multiple faults in a PV array. The BHAM network can store the training patterns in a connecting matrix when modeling the human cognitive process [17]. Its mechanism investigates various conditions represented by encoding weighted values in a weighting matrix; this process can maximize information representation and reduce memory requirements. In addition, BHAM is able to learn and recall various types of input and output associations. A traditional BHAM network can associate pairs of patterns in two directions. Its model does not have a hidden layer or nonlinear processing layer. Thus, it is difficult to solve nonlinear separable problems. In this study, the proposed modified configuration with the hidden layer (Gaussian functions) is used to solve nonlinear separable problems [14], and its manner can deal with inputs and outputs using binary data $(0 / 1)$ or numerical data $(0,1,2,3, \ldots)$, as seen in Figure 1 . Using nonlinear output feedback, it can also act using biological behavior in the form of hetero-associative memories for different data types and data lengths. The indexes are used to screen the degradation levels of input powers, $p_{1}, p_{2}, p_{3}, \ldots$. Then, the operation state of each PV panel can be identified. The proposed BHAM is designed as a classifier to locate the multiple faults in a PV array that can indicate one or more PV panel faults. In contrast to traditional machine learning methods, the BHAM network can reduce the computation time and memory storage requirements. The proposed model is also easily implemented in an embedded system or a tablet PC. Thus, experimental results show it is capable of detecting multiple faults in a rooftop PV system.

The remainder of this paper is organized as follows. Section 2 describes the methodology, including the maximum output power estimation, typical fault analysis in a PV array, and the BHAM network. Sections 3 and 4 present the simulation results and conclusion to demonstrate the efficiency of the proposed model in locating multiple faults. 


\section{Methodology}

\subsection{Maximum Output Power Estimation and Fault Feature Extraction}

PV panels are wired in a parallel or series configuration to form a PV array and increase output voltage or current (12/48 voltage). When wiring PV panels in a parallel configuration, the output amperage (current) is additive, $I=q \times I_{q}$, where $q$ is the number of PV panels in parallel, as seen in Figure 2a. The output voltage, $\mathrm{V}$, and current, I, for $q$ PV panels in a parallel or series configuration can be expressed as:

Parallel:

$$
I=q \times I_{q} \approx \frac{a \times I_{L}}{\eta}
$$

Series:

$$
V=s \times V_{q}
$$

where $I_{L}$ is the grid rated load current; parameter, $a$, is the environment modified factor, and $a=7.8-9.0$ in Taiwan; $\eta$ is the output effectiveness; $V_{q}$ is the rated voltage per PV panel, $V_{q}=12$ or 24 voltage; and $s$ is the number of PV panels in a series. Thus, the output power can be increased, as:

$$
P=V \times I=q \times s \times V_{q} \times I_{q}
$$

(a)
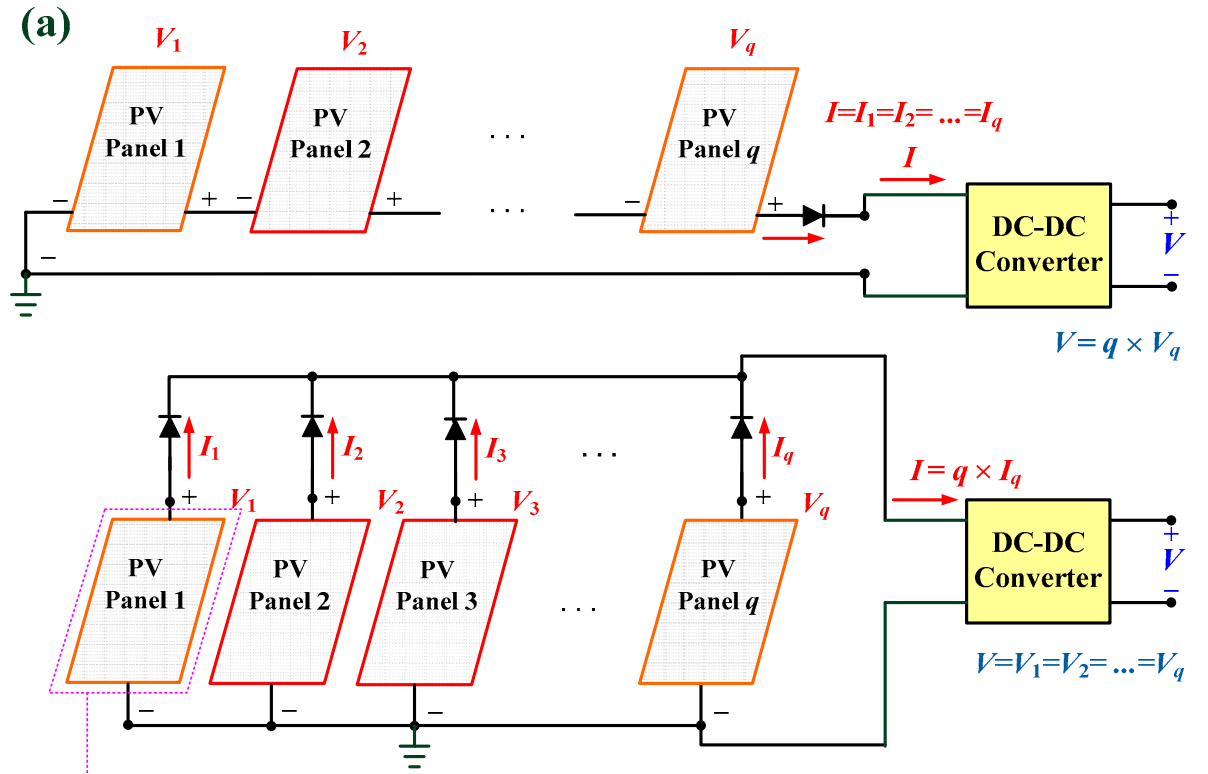

(b)

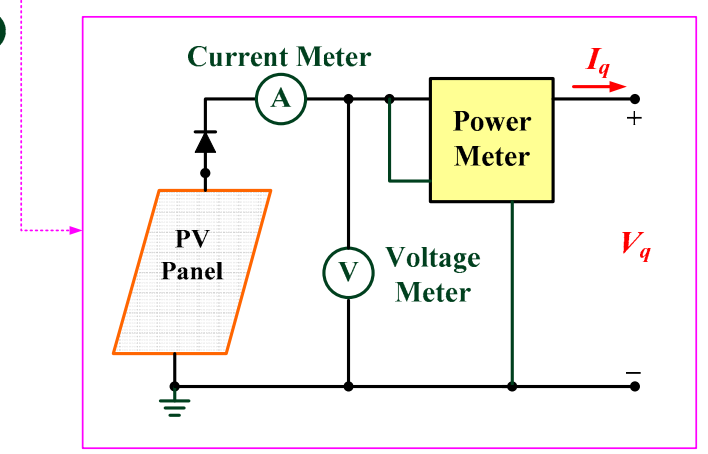

Figure 2. Schematic diagram of PV array. (a) PV array in a parallel or series configuration, (b) Measurement system of each PV panel. 
A MPPT algorithm, such as the incremental conductance method (ICM) [1,2] or ICM-based method [3], is used to estimate the output power of each PV panel. If we suppose that the PV panels are wired in a parallel configuration, as seen in Figure 2a, and that the electrical characteristics of the PV panels are identical, the estimated terminal voltage, $V_{q, e s t}$, and photo-current, $I_{p h, q}$, of each PV panel are as follows:

$$
\begin{gathered}
V_{q, e s t}=\frac{n_{s} b T A}{Q} \ln \left(\frac{n_{p} I_{p h}+n_{p} I_{s a t}-I_{q}}{n_{p} I_{s a t}}\right) \\
I_{p h, q}=\left[I_{s c}+k_{s c}\left(T_{c}-T_{r}\right)\right] \frac{S}{100} \\
V_{\text {est }}=V_{q, e s t}+\rho \times\left(T_{c}-25\right)
\end{gathered}
$$

where $I_{\text {sat }}$ is the reverse saturation current; $T$ is the surface temperature of the cell; $n_{p}$ and $n_{s}$ are the number of modules connected in parallel and series, respectively; $Q$ is the electron charge; $b$ is Boltzmann's constant; $A$ is the $\mathrm{p}-\mathrm{n}$ junction ideality factor, which is typically in the range of $1<A<5$ for different manufacturers; $T_{c}$ is the ambient temperature; $T_{r}$ is the reference temperature $\left(25^{\circ} \mathrm{C}\right)$; $I_{s c}$ is the cell short-circuit current at $T_{r} ; k_{s c}$ is the short-circuit current temperature coefficient; $S$ is the solar radiation; $\rho$ is the temperature compensation coefficient. Temperature and irradiance sensors are required in each PV array (in a parallel or series configuration).

Therefore, the maximum output power (MOP) of each PV panel, $P_{q, e s t}$, and total MOP, $P_{\text {est }}$, can be estimated as:

$$
\begin{gathered}
P_{q, e s t}=V_{q, e s t} \times I_{p h, q} \\
P_{e s t}=q \times P_{q, e s t}
\end{gathered}
$$

The PV characteristics, such as power-voltage (P-V) and current-voltage (I-V) curves, vary widely depending on atmospheric conditions, such as solar radiation and temperature. Fault voltages, fault currents, and output power degradations are affected by fault types. Typical faults occurring in a PV panel include: (a) open circuit fault (OCF); (b) bridged (line-to-line) fault (BF); (c) and (d) lower and upper grounded faults, respectively, (LGF/UGF) $[6,11,12]$. When any fault occurs in a PV panel, voltage, current, and power meters can be employed to measure the output PV power, $P_{q, \text { mea }}$, of each panel, as seen in the measurement system in Figure $2 b$.

The MPPT algorithm can be used to estimate the MOP. It is known that when measuring output power degradation, the measured value and estimated value are not identical for given environmental conditions [19]. Hence, the MOP is an important index from the viewpoint of fault identification. For each PV panel, this power degradation index can be defined as:

$$
p_{q}=P_{q, \text { mea }} / P_{q, e s t}
$$

As shown in Table 1, it can be seen that the index, $p_{q}$, of power degradations is computed in per-unit $(\mathrm{pu})$ quantities with an interval of $[0,1]$ and with an estimated output power, $P_{\text {qrest }}$, and a measured output power, $P_{q, \text { mea }}$, at each PV panel. According to the power degradation of each PV panel, the index can be parameterized with certainty factors as $[6,11]$

$$
\begin{aligned}
& \mu_{q 1}=\left\{\begin{array}{l}
1,0.90 \leq p_{q} \leq 1.00 \\
\exp \left(-1 \times\left(\frac{p_{q}-0.90}{\sigma_{q}}\right)^{2}\right), p_{q}<0.90
\end{array}\right. \\
& \mu_{q 2}=\left\{\begin{array}{l}
1,0.00 \leq p_{q} \leq 0.80 \\
\exp \left(-1 \times\left(\frac{p_{q}-0.80}{\sigma_{q}}\right)^{2}\right), p_{q}>0.80
\end{array},\right.
\end{aligned}
$$

where $\sigma_{q}=0.10$; $n$ the identical PV panels are connected in a parallel or series configuration, $q=1,2,3$, $\ldots, n\left(n=8\right.$ means 8 PV panels in this study); $p_{q}$ is the power degradation index. The functions of 
certainty factors for normal condition and fault types are shown in Figure 3. The operation state of each PV panel can be identified as:

$$
s_{q}=\left\{\begin{array}{ll}
1, & \mu_{q 2}>\mu_{q 1} \\
0, & \mu_{q 2} \leq \mu_{q 1}
\end{array} .\right.
$$

The state vector $S=\left[s_{1}, s_{2}, \ldots, s_{q}, \ldots, s_{n}\right]=[0 / 1,0 / 1, \ldots, 0 / 1, \ldots, 0 / 1]$. Equation (12) is the generalized form to screen the operation state of each PV panel for large PV arrays. For $n$-digit binary numbers, each bit has two states, $\{0,1\}$, and the total number of the $n$-digit binary string is $2^{n}$ combinations. In this study, with $8 \mathrm{PV}$ panels in a series configuration $\left(n=8, q^{\prime}=0,1,2, \ldots, n\right)$, we have $2^{8}=256$ combinations of different binary patterns, as:

$$
\begin{aligned}
& \left(\begin{array}{c}
8 \\
q^{\prime}
\end{array}\right)=\frac{8 !}{q \prime !\left(8-q^{\prime}\right) !},\left(\begin{array}{l}
8 \\
0
\end{array}\right)=\left(\begin{array}{l}
8 \\
8
\end{array}\right)=1 \\
& N_{q^{\prime}}=\sum_{q^{\prime}=0}^{n}\left(\begin{array}{c}
n \\
q^{\prime}
\end{array}\right)=\sum_{q^{\prime}=0}^{n} \frac{8 !}{\left(q^{\prime} !\right)\left(8-q^{\prime}\right) !}=256
\end{aligned}
$$

Therefore, we have 256 binary patterns to represent the fault patterns. The corresponding binary patterns can be encoded as binary values of 1 or 0 , with the value " 1 " indicating a "PV fault", and everything else can be encoded by the value " 0 ", as seen input patterns in Figure 4 . A bidirectional associative memory network can thus be designed as a neuro-dynamic model to identify the faults within a PV array.

Table 1. The output power degradation for different fault types $[6,11]$.

\begin{tabular}{lc}
\hline \multicolumn{1}{c}{ Fault Type } & Power Degradation Index, $\boldsymbol{p}_{\boldsymbol{q}}$ \\
\hline 1. Normal (Nor) & $<0.10$ \\
2. Lower Grounded Fault (LGF) & $0.10 \sim 0.30$ \\
3. Mismatch Fault/Hot Spot (MF/HS) & $0.30 \sim 0.60$ \\
4. Bridged (line-o-line) Fault (BF) & $0.30 \sim 0.50$ \\
5. Open Circuit Fault (OCF) & 0.00 \\
6. Upper Grounded Fault (UGF) & $>0.60$ \\
\hline
\end{tabular}

$\triangle$ Normal Condition $\quad \square$ Fault Event

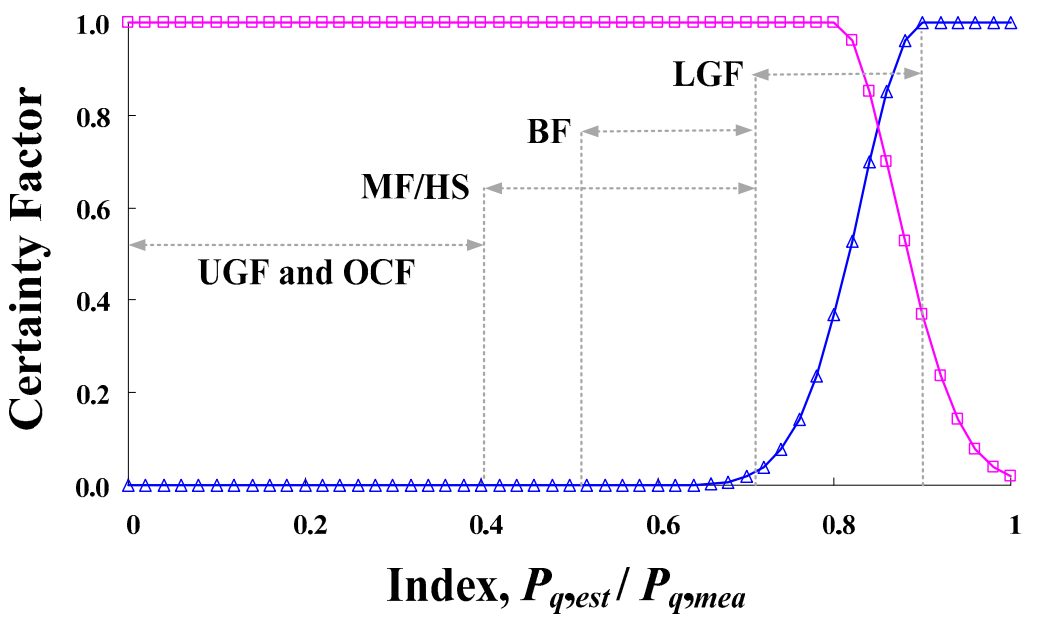

Figure 3. The certainty factors versus the index, $p_{q}$, for normal condition and fault types. 

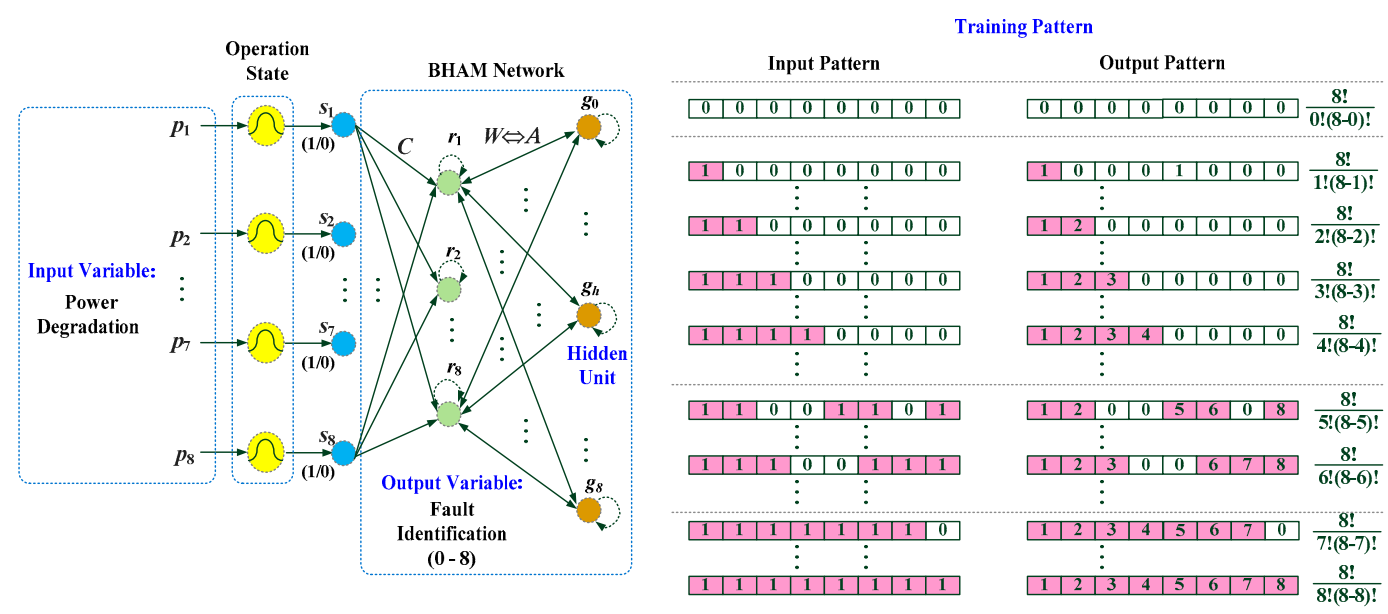

Figure 4. The configuration of BHAM network and input-output paired training patterns.

\subsection{Bidirectional Associative Memory Network}

A recurrent memory network is an unsupervised dynamic learning system and can be classified into either auto-associative or hetero-associative memory models. A BHAM network is a feedback pattern mechanism that allows for the generation of new patterns, noise filtering, and pattern completion $[13,15]$. It has a network that contains an input layer, output layer, and network connections, as shown in Figure 4. The network connections between the process units are bidirectional and in a loop configuration; this dynamic system can deal with binary data $(0 / 1)$ and numerical data $(0,1,2$, $\ldots, 8)$, and can also represent biological behavior with both auto-associative and hetero-associative memories. Its multi-layer mechanism can generate output patterns using nonlinear output feedback, such as Gaussian functions, and can also associate different data types (numerical data) or data lengths (output vector). The machine-learning algorithm stores information and matrices using noise-free versions of the input and output patterns. In this study, the BHAM is used to associate the power degradation indexes (as input variables), and then indicate the multiple faulted PV panels (as output variables). Given 8 PV panels in an array, the BHAM algorithm has two stages, the learning stage and the recalling stage, as delineated below.

\section{Learning stage}

Step (1) establish the input-output pair of the training pattern, $S_{k}$ and $R_{k}, k=1,2,3, \ldots, K, K=256$ binary training patterns, where state vector, $S_{k}=\left[s_{k 1}, \ldots, s_{k i}, \ldots, s_{k n}\right]^{\mathrm{t}}, s_{k i} \in\{0,1\}$, and the fault pattern, $R_{k}=\left[r_{k 1}, \ldots, r_{k j}, \ldots, r_{k n}\right]^{\mathrm{t}}, R_{k j} \in\{0,1\}$.

Step (2) establish the connecting matrix $C$ using $K$ pairs of training patterns,

$$
C=\sum_{\mathrm{k}=1}^{K} S_{k}{ }^{t} R_{k}
$$

where $C=\left[w_{i j}\right]_{n \times n}$.

Step (3) find $n$ eigenvalues, $\lambda_{i}=w_{i j}, i=j=1,2,3, \ldots, 8$, the weight matrix, $W$, and the associative matrix, $A$, for the eight faults as follows:

$$
W \Leftrightarrow A
$$




$$
\begin{gathered}
W=\left[\begin{array}{cccccc}
\omega_{0} \times \lambda_{1} & \omega_{0} \times \lambda_{2} & \cdots & \omega_{0} \times \lambda_{5} & \cdots & \omega_{0} \times \lambda_{8} \\
\omega_{1} \times \lambda_{1} & \omega_{1} \times \lambda_{2} & \cdots & \omega_{1} \times \lambda_{5} & \cdots & \omega_{1} \times \lambda_{8} \\
\vdots & \vdots & \ddots & \vdots & \ddots & \vdots \\
\omega_{5} \times \lambda_{1} & \omega_{5} \times \lambda_{2} & \cdots & \omega_{5} \times \lambda_{5} & \cdots & \omega_{5} \times \lambda_{8} \\
\vdots & \vdots & \ddots & \vdots & \ddots & \vdots \\
\omega_{8} \times \lambda_{1} & \omega_{8} \times \lambda & \cdots & \omega_{8} \times \lambda_{5} & \cdots & \omega_{8} \times \lambda_{8}
\end{array}\right] \\
A=\left[\begin{array}{cccccccc}
0 & 0 & 0 & 0 & 0 & 0 & 0 & 0 \\
1 & 0 & 0 & 0 & 0 & 0 & 0 & 0 \\
\vdots & \vdots & \vdots & \vdots & \vdots & \vdots & \vdots & \vdots \\
1 & 2 & 3 & 4 & 0 & 0 & 0 & 0 \\
\vdots & \vdots & \vdots & \vdots & \vdots & \vdots & \vdots & \vdots \\
1 & 2 & 3 & 4 & 5 & 6 & 7 & 8
\end{array}\right],
\end{gathered}
$$

where $\omega_{h}, h=0,1,2, \ldots, 8$, is the weight value for normal condition and eight faults within the weight matrix, $W=\left[\omega_{h i}\right]_{9 \times 8}, \omega_{h i}=\omega_{h} \times \lambda_{i}$, and each element in the associative matrix, $A=\left[a_{h j}\right]_{9 \times 8}$, element, $a_{h j}$, is encoded as numerical data from value " 1 " to value " 8 " to indicate which one is fault in a PV array, and the normal condition is encoded as the value " 0. "

\section{Recalling stage}

Step (1) obtain the network connecting matrices, $C, W$, and $A$, and apply the testing pattern $S_{0}=\left[s_{1}, s_{2}, \ldots, s_{i}, \ldots, s_{8}\right]$ to the BHAM network;

Step (2) associate the output pattern, $R_{0}=\left[r_{1}, r_{2}, \ldots, r_{i}, \ldots, r_{8}\right]^{\mathrm{T}}$, as:

$$
R_{0}=C^{T} S_{0}, r_{i}=\sum_{i=1}^{8} w_{j i} s_{i}, i=1,2,3, \ldots, 8
$$

Step (3) transit the output pattern, $R_{0}$, to the Gaussian function unit, $g_{h}$, and the output of $g_{h}$, as:

$$
\begin{gathered}
g_{h}=\exp \left(\frac{-1}{2 \sigma^{2}} \times\left(E D_{h}\right)^{2}\right), h=0,2,3, \ldots, 8 \\
E D_{h}=\sqrt{\sum_{i=1}^{8}\left(\omega_{h i}-r_{i}\right)^{2}}
\end{gathered}
$$

where $\sigma$ is the standard deviation, $\sigma=\min \left(E D_{h}\right) \times 0.90$; if the pattern, $R_{0}$, is similar to any row weight vector of matrix $W$, the $E D_{h}$ will be small $\left(E D_{h} \rightarrow 0\right.$, argmin ||$\left.E D_{h}||, h=0,2,3, \ldots, 8\right)$ and the $g_{h}$ unit will approach 1 . The $g_{h}$ unit is the index that measure the similarity degree among nine row weight vectors.

Step (4) transit the outputs of the $g_{h}$ units to the $r_{i}$ unit with nonlinear feedback and compute the output of the $r_{i}$ unit using the hard limit function with a threshold value of 0.50 , as:

$$
\begin{gathered}
g_{h}^{\prime}=\left\{\begin{array}{l}
1, g_{h} \geq 0.50 \\
0, g_{h}<0.50
\end{array}\right. \\
R_{0 p}=\left[r_{1}, r_{2}, \ldots, r_{i}, \ldots, r_{8}\right], r_{i}=\sum_{h=1}^{9} a_{i h} g_{h}^{\prime}, i=1,2,3, \ldots, 8
\end{gathered}
$$

Step (5) transit bidirectional patterns repeatedly between the $r_{i}$ units and $g_{h}$ units until bidirectional stability is reached. When the patterns, $R_{0}$, is not changed, and $\Delta R_{0 p}=\left\|R_{0 p}-R_{0 p-1}\right\|=0$, where $p$ is the iteration number, then the BHAM algorithm will be terminated. 
When the BHAM network reaches bidirectional stability, the state $s_{i}=1$, means one or more faults have been detected. Thus, the BHAM network associates the numerical data to locate which PV panel is at fault within the PV array. A flow chart of the BHAM algorithm for multiple fault location is shown in Figure 5.

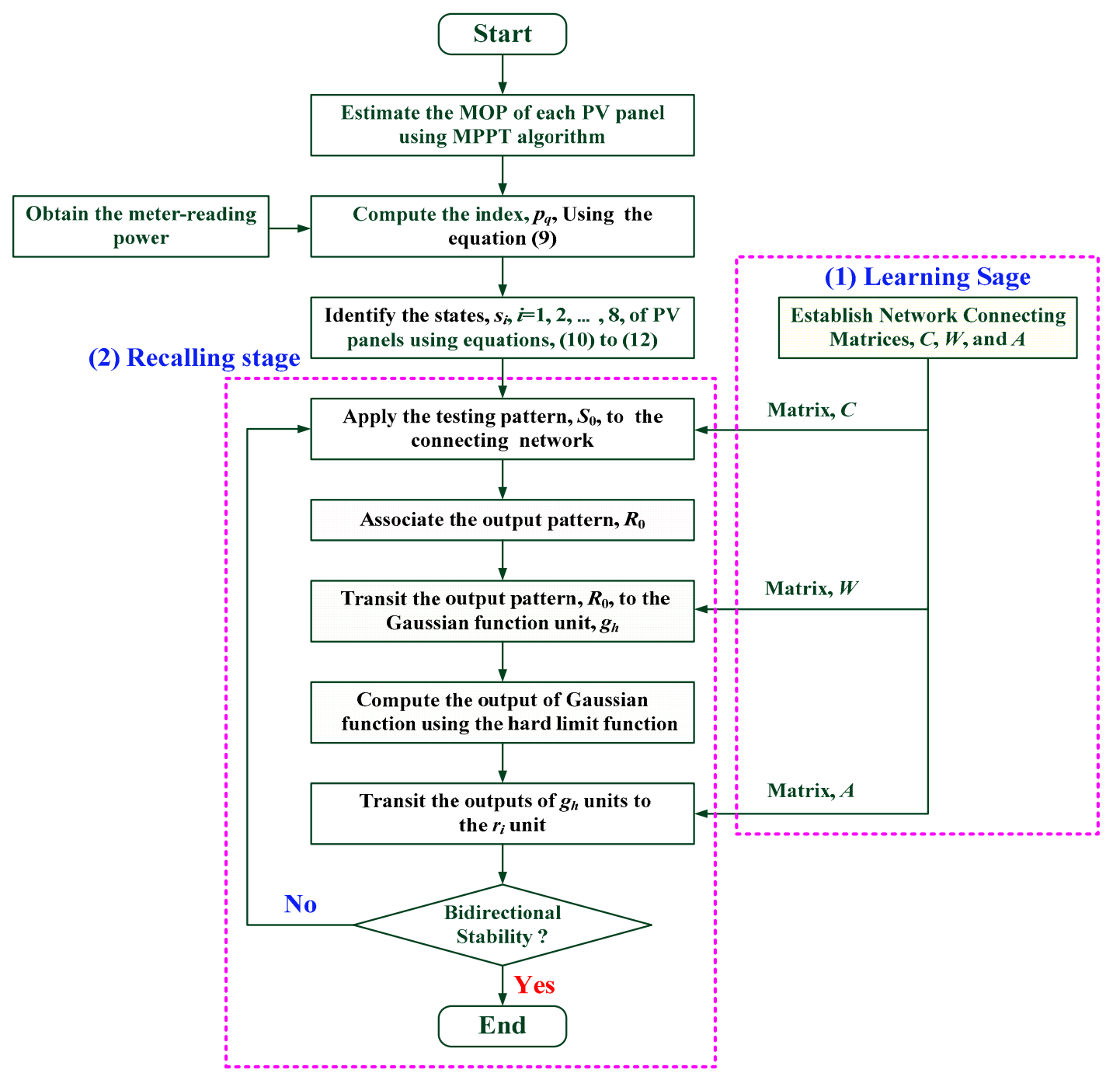

Figure 5. The flow chart of fault location and BHAM algorithm.

\section{Experimental Results and Discussion}

The proposed multiple fault location algorithm using a BHAM network was designed and tested on a PC Pentium-IV 2.4 GHz with 480 MB RAM and MATLAB mathematical computing software (MathWorks, Natick, Massachusetts, USA). Relevant specific parameters of each PV panel used in this study are shown in Table 2. In Taiwan's subtropical outdoor environment, the average solar radiation and temperature are approximately $0.20-0.80 \mathrm{~kW} / \mathrm{m}^{2}$ and $30-40{ }^{\circ} \mathrm{C}$, respectively, during the summer season. For various radiation and temperature values, the MPPT algorithm [1-3,19-21] can be employed to control the DC-DC boost converter until the desired MOP and output voltage is reached. In this study, an ICM-based method [1,3] is used to estimate the desired output and adjust the boost converter's duty ratio to match the maximum point. The MPPT is used to track the MOP by adjusting the voltage as solar radiation and temperature increase from $0.2 \mathrm{~kW} / \mathrm{m}^{2}$ to $1.0 \mathrm{~kW} / \mathrm{m}^{2}$ and from $30{ }^{\circ} \mathrm{C}$ to $45^{\circ} \mathrm{C}$, respectively. The $\mathrm{P}-\mathrm{V}$ and the $\mathrm{I}-\mathrm{V}$ characteristic curves of each PV panel are shown in Figure 6. Hence, when atmospheric conditions change, the MPPT algorithm takes less 
than 10 switching controls to achieve the desired value. During the summer season, each PV panel had output powers of $199.1 \mathrm{~W}$ to $262.2 \mathrm{~W}$, output currents of $9.8 \mathrm{~A}$ to $13.6 \mathrm{~A}$, and an output voltage of $19.8 \mathrm{~V}$, as seen in Figure $6 \mathrm{a}, \mathrm{b}$. The boost converter adjusts the duty ratio until the desired values of output power and voltage are reached, as seen in Figure $6 \mathrm{c}$. The experimental results confirm that the MPPT can estimate the output power of each panel under various atmospheric conditions, and it can also work during clear day, low solar radiation and at the night-to-day transition.

Table 2. Specific parameters of each PV panel (at solar radiation of $1.0 \mathrm{~kW} / \mathrm{m}^{2}$ and a temperature of $\left.25^{\circ} \mathrm{C}\right)[1,3]$.

\begin{tabular}{cc}
\hline Specific Parameter & Value \\
\hline Maximum Power $P_{\max }$ & $87.70(\mathrm{~W})$ \\
Short-circuit Current $I_{S C}$ & $4.80(\mathrm{~A})$ \\
Open-circuit Voltage $V_{O C}$ & $21.70(\mathrm{~V})$ \\
Rated Voltage $V_{R}$ & $19.14(\mathrm{~V})$ \\
Rated Current $I_{R}$ & $4.58(\mathrm{~A})$ \\
Number of Modules Connected in Series $n_{S}$ & 36 \\
Number of Modules Connected in Parallel $n_{p}$ & 2 \\
\hline
\end{tabular}

For a rooftop PV system, we built an array of about 3.2-4.2 kW PV in the summer season, which consists of two strings with 16 identical PV panels in parallel (the same manufacturer), as shown in the experimental setup in Figure 7a. The specific parameters of the rooftop PV system with two strings (1\# and 2\#) in the summer season are shown in Table 3. The measurement system includes voltage, current, and power collected through data acquisition meters and communication lines. Two BHAM networks are employed to locate PV panels with faults. The times between 8:00 a.m. and 18:00 p.m. was divided into 40 time slots ( $15 \mathrm{~min}$ time slots) for monitoring the rooftop PV system. Atmospheric conditions are shown in Figure $7 \mathrm{~b}$, and each of the PV panels, string measurements, and estimation powers are shown in Figure 7c. The total string output power was 2.87-3.44 kW, and the output power of each panel was $0.18-0.23 \mathrm{~kW}$. In addition, each BHAM network had eight input nodes, nine hidden nodes, and eight output nodes for detecting fault in each string. The total memory storage for the connecting matrix, $C(8 \times 8)$, weight matrix, $W(8 \times 9)$, and associative matrix, $A(8 \times 9)$, is 832 bytes $(208 \times 4$ bytes). The weight matrix, $W$, and associative matrix, $A$, are:

$$
\begin{aligned}
& W \Leftrightarrow A \\
& {\left[\begin{array}{ccccc}
0.0 & 0.0 & \cdots & \cdots & 0.0 \\
1.0 \times \lambda_{1} & 1.0 \times \lambda_{1} & \cdots & \cdots & 1.0 \times \lambda_{1} \\
1.5 \times \lambda_{2} & 1.5 \times \lambda_{2} & \cdots & \cdots & 1.5 \times \lambda_{2} \\
2.0 \times \lambda_{3} & 2.0 \times \lambda_{3} & \cdots & \cdots & 2.0 \times \lambda_{3} \\
\vdots & \vdots & \ddots & \ddots & \vdots \\
4.5 \times \lambda_{8} & 4.5 \times \lambda_{8} & \cdots & \cdots & 4.5 \times \lambda_{8}
\end{array}\right] \Leftrightarrow\left[\begin{array}{ccccc}
0 & 0 & 0 & \cdots & 0 \\
1 & 0 & 0 & \cdots & 0 \\
1 & 2 & 0 & \cdots & 0 \\
1 & 2 & 3 & \cdots & 0 \\
\vdots & \vdots & \vdots & \ddots & \vdots \\
1 & 2 & 3 & \cdots & 8
\end{array}\right]}
\end{aligned}
$$

where elements, $\lambda_{1}=\lambda_{2}=\ldots=\lambda_{8}=128$, are eigenvalues of matrix $C$.

Table 3. Specific parameters of a rooftop PV system with two strings in summer season.

\begin{tabular}{cccccc}
\hline String & $\begin{array}{c}\text { Panel } \\
\text { Number }\end{array}$ & $\begin{array}{c}\text { Radiation } \\
\mathbf{k W} / \mathbf{m}^{\mathbf{2}}\end{array}$ & Temperature $^{\circ} \mathbf{C}$ & $\begin{array}{c}\text { Each Panel } \\
\text { Power (W) }\end{array}$ & $\begin{array}{c}\text { Total Output Power } \\
(\mathbf{k W}) \text { and Current (A) }\end{array}$ \\
\hline $1 \#$ & 8 & $0.2-1.0$ & $30-45$ & $199.1-262.2$ & $\begin{array}{r}1.6-2.1 \mathrm{~kW} \\
78.4-108.8 \mathrm{~A}\end{array}$ \\
\hline $2 \#$ & 8 & $0.2-1.0$ & $30-45$ & $199.1-262.2$ & $\begin{array}{c}1.6-2.1 \mathrm{~kW} \\
78.4-108.8 \mathrm{~A}\end{array}$ \\
\hline
\end{tabular}


(a)

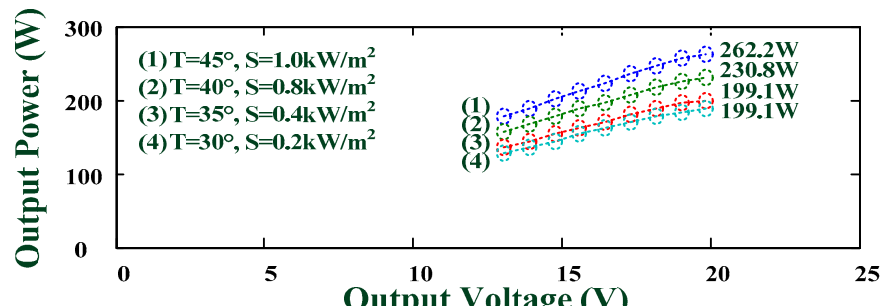

(b)

Output Voltage (V)

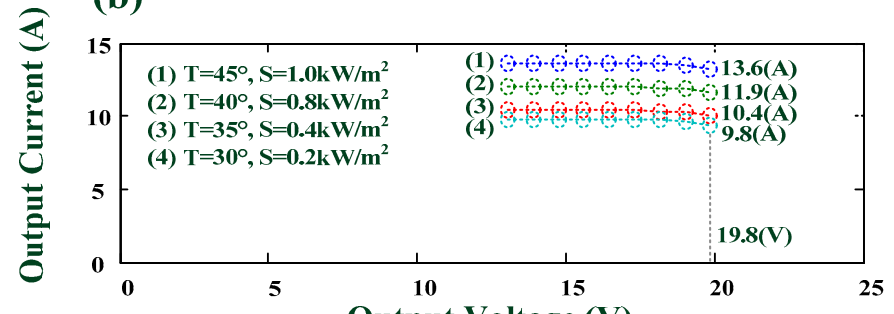

(c) Output Voltage (V)

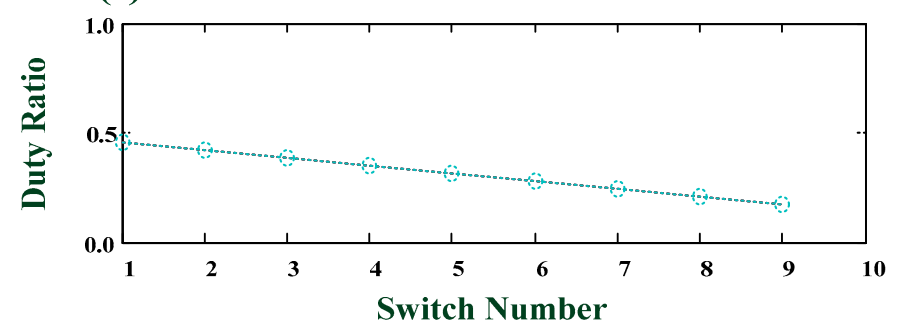

Figure 6. The MPPT estimated results. (a) Output power versus output voltage, (b) Output current versus output voltage, (c) Duty ratio versus switch number.

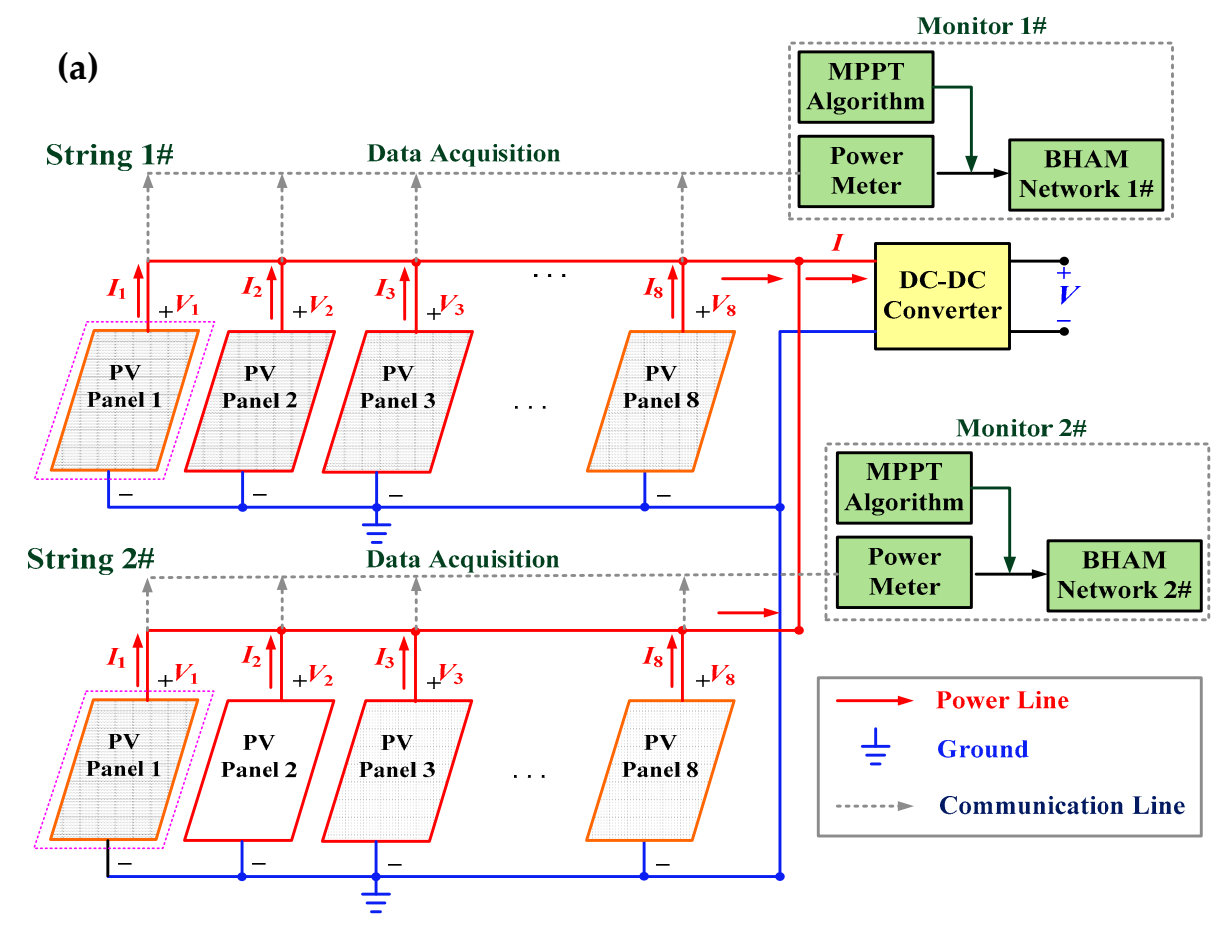

Figure 7. Cont. 
(b)

(c)

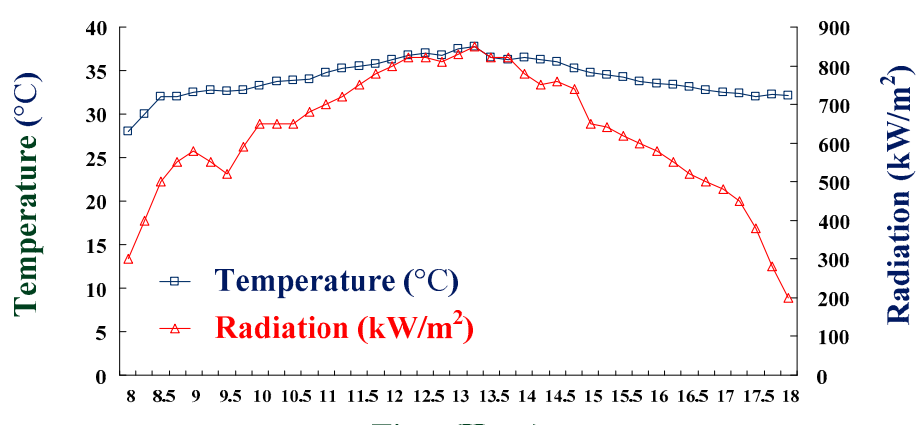

Time (Hour)

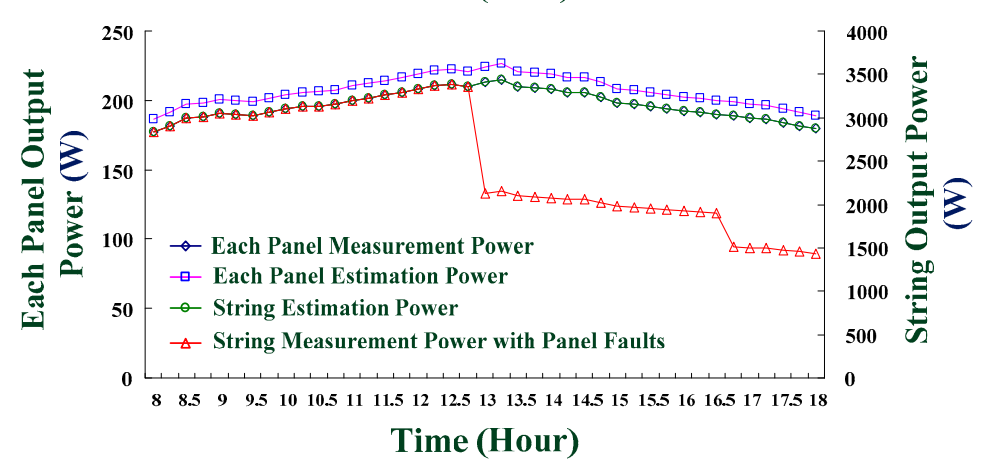

Figure 7. Experimental setup. (a) Schematic diagram of a rooftop PV system, consisting of 2 strings with 16 PV panels; (b) Solar radiation and temperature versus time (from 8:00 a.m. to 18:00 p.m.); (c) Each PV panel and string measurement power and estimation power.

If multiple open circuit faults occurred in PV panels, 1\#, 2\#, and 3\# at string 1\#, and in PV panels, 1\#, 2\#, and 3\# at string 2\#; therefore output power was not generated. At 13:00, solar radiation and temperature were $830 \mathrm{~kW} / \mathrm{m}^{2}$ and $37.5^{\circ} \mathrm{C}$; the MPPT algorithm was employed to estimate the MOP, 224.4 W, for each PV panel, and the output power for each faulted panel was $0.0 \mathrm{~W}$. Six of the PV panels in parallel were disconnected, and thus, the string output power degraded $37.5 \%$ of the MOP, and an overcurrent was not generated. Hence, 16 power indexes could be estimated using Equation (9). The fault location procedures at the recall stage for two BHAM networks are as follows:

Step (1) String 1\#: $\left[p_{1}, p_{2}, p_{3}, p_{4}, p_{5}, p_{6}, p_{7}, p_{8}\right]=[0.00,0.00,0.00,0.95,0.96,0.95,0.96,0.97]$;

String 2\#: $\left[p_{1}, p_{2}, p_{3}, p_{4}, p_{5}, p_{6}, p_{7}, p_{8}\right]=[0.00,0.00,0.00,0.96,0.97,0.96,0.96,0.95]$;

Step (2) power indexes were parameterized using Equations (10) to (12). The operation states of $16 \mathrm{PV}$ panels were identified as

String 1\#: [s1, s2, s3, s4, s5, s6, s7, s8] = [1, 1, 1, 0, 0, 0, 0, 0];

String 2\#: [s1, s2, s3, s4, s5, s6, s7, s8] = [1, 1, 1, 0, 0, 0, 0, 0];

Step (3) associated the output patterns: String 1\#: [r1, r2, r3, r4, r5, r6, r7, r8] = [256, 256, 256, 192, 192, 192, 192, 192];

String 2\#: [r1, r2, r3, r4, r5, r6, r7, r8] = [256, 256, 256, 192, 192, 192, 192, 192];

Step (4) computed the outputs of Gaussian function units:

String 1\#: [g1, g2, g3, g4, g5, g6, g7, g8] = [0.0454, 0.2665, 0.5738, 0.4881, 0.2148, 0.0888, 0.0362, 0.0147, 0.0060];

String 2\#: [g1, g2, g3, g4, g5, g6, g7, g8] = [0.0454, 0.2665, 0.5738, 0.4881, 0.2148, 0.0888, 0.0362, 0.0147, 0.0060]; 
Step (5) transited the outputs of Gaussian function units to the output units using Equations (22) and (23):

$$
\begin{aligned}
& \text { String 1\#: [r1, r2, r3, r4, r5, r6, r7, r8] = [1, 2, 3, 0, 0, 0, 0, 0]; } \\
& \text { String 2\#: }[\mathrm{r} 1, \mathrm{r} 2, \mathrm{r} 3, \mathrm{r} 4, \mathrm{r} 5, \mathrm{r6}, \mathrm{r} 7, \mathrm{r} 8]=[1,2,3,0,0,0,0,0]
\end{aligned}
$$

Step (6) reached bidirectional stability and terminated the BHAM algorithm.

In addition, from 8:00 a.m. to 18:00 p.m., 41 experimental tests were conducted, and the results of fault locations indicated normal operating conditions from 8:00 a.m. to 12:45 p.m., 6 PV panels with faults between 13:00 p.m. and 16:30 p.m., and 8 PV panels with faults from 16:45 p.m. to 18:00 p.m. The experimental results indicate a fault location accuracy of $100 \%$ (hit rate). Experimental results confirmed that the proposed model enables accurate multiple fault location within the PV array.

Therefore, by using electrical examinations, these promising results could be given to operators prior to performing visual inspections using thermal sensors or IRTs. In solar panel maintenance, visual inspections could thus be conducted to check for defects in any of the panels. In addition, drone/light unmanned vehicle techniques with infrared cameras could also be deployed instead of ground-based inspection techniques [21-23]. Furthermore, pilot and sensor operator inspected visual images can be taken from a height of 10-15 m during maintenance in a large-scaled PV system or rooftop system, which could reduce the number of manual tasks required, and reduce the amount of time taken to conduct inspections. Therefore, maintenance engineers could visit fault locations without the need to survey the entire PV array, which could ultimately reduce operating costs. Faults types could be rapidly examined using manual and drone inspections, which would reduce the need for online examinations. Electrical examinations and unmanned technology could thus be integrated into inspections, thereby reducing costs and time.

To compare the proposed machine-learning model with other intelligent methods, we used the same 256 input-output pairs of training data to train an artificial neural network, where 256 training patterns were used to determine the configuration of probability neural network (PNN) [24]. The multi-layer PNN consists of an input layer with 8 input nodes (independent variables), a pattern layer with 256 nodes, a summation layer with 9 nodes, and an output layer with 8 nodes (dependent variables). Optimal PNN parameters were determined using the traditional optimization algorithms, such as least-square algorithm or the gradient descent algorithm [25-27], which iteratively analyzes the mean squared error and adjust the optimal parameter to minimize the objective function. Under the initial condition $\sigma=1.0000$, and the pre-specified tolerance mean squared error, $\leq 10^{-2}$, the iteration computing process took about $<100$ iterative computations to reach the convergent condition, as seen in Figure 8. Optimal smoothing parameter, 0.1125 and 0.0773 , was obtained to minimize the mean squared error, and improve accuracy rate with learning rates, 0.5 and 0.6. However, initial condition assignments, such as initial network parameters, learning rates, and pre-specified tolerance value, can affect the model's learning outcome performance. For example, a greater learning rate, $>0.6$, allowed rapid learning stage to reach the convergent condition. However, its computation easily trapped in the local minimum and perturbation around the desired optimal parameters, or tended to the divergent condition. With learning rate, $0.5-0.6$, and tolerance value, $<10^{-2}$, the learning stage could guarantee to reach the convergent condition in $<50$ iteration computations. In addition, fill-in with elements in the input and output matrices can increase computing time, memory storage requirements, and iterative computations. Therefore, considering 4 bytes for digital storage, the total memory storage was 16,384 bytes (input matrix: $256 \times 8 \times 4$ bytes and output matrix: $256 \times 8 \times 4$ bytes). PNN also provided a $100 \%$ hit rate and provided promising results for detecting multiple fault locations. However, in contrast to the proposed model, the iterative computing processes and memory storage requirements had two limitations.

For the input-output paired training patterns, we had 256 combinations of different possible events for 8 inputs versus 8 outputs, and also had 9 possible events, including normal condition and 8 faulted events, as shown in Figure 4. Thus, the BHAM topological size could be kept in a 
fixed configuration, such as the 8-8-9 topology used in this study. The proposed model showed very fast training and recalling processes; however, its memory storage requirement needs to be reduced, as shown in Table 4 . The dimensions of the training patterns and the memory storage needs could be reduced from 16,384 bytes to 832 bytes, overhead than the PNN method. The proposed BHAM network could directly deal with the binary input pattern, and then act to associate the output pattern, as corresponding output pattern with numerical data to identify the possible faulted PV panel. In the recalling stage, the BHAM network took an average time of $<0.03 \mathrm{~s}$ and $\leq 2$ iterative computations to reach bidirectional stability, as seen in Figure 8. Therefore, the proposed model could be easy to implement in portable device designs, such as intelligent mobile vehicles or drone/light unmanned vehicles. The measurement system transited metering PV panels' output powers to a mobile vehicle via WiFi wireless communication (IEEE 802.11 standards wireless local area network, WLAN) [28]. Thus, the reliability of location and detection results could be enhanced.

Table 4. Comparison of the proposed model and multi-layer PNN method.

\begin{tabular}{|c|c|c|}
\hline Task & BHAM Network & PNN Method [24,25] \\
\hline Network Configuration & $8-8-9$ & $8-256-9-8$ \\
\hline Number & 2 BHAM networks for 2 strings & 2 PNNs for 2 strings \\
\hline Training Data & 256 input-output pairs & 256 input-output pairs \\
\hline Storage Matrix & $C_{8 \times 8}, W_{9 \times 8}$, and $A_{9 \times 8}$ & Input $(256 \times 8)$ and output $(256 \times 8)$ matrices \\
\hline Memory Storage & 832 bytes & 16,384 bytes \\
\hline Process Unit & $\begin{array}{l}\text { Gaussian function and } \\
\text { hard limit function }\end{array}$ & Gaussian function \\
\hline Learning Algorithm & Bidirectional associative memory & Least square algorithm \\
\hline Learning Stage & $\begin{array}{c}\text { Establish matrices, } \\
C, W \text {, and } A \\
\text { (Matrix Operation) }\end{array}$ & Iteration computing process $<100$ \\
\hline Recalling Stage & Iteration computing process $\leq 2$ & - \\
\hline Execution Time & Average time: $<0.03 \mathrm{~s}$ & Average time: $<20 \mathrm{~s}$ \\
\hline Testing Pattern & 256 patterns for each BHAM network & 256 patterns for each PNN \\
\hline Accuracy & $100 \%$ & $100 \%$ \\
\hline Application & $\begin{array}{c}\text { Easy to implement } \\
\text { in a mobile intelligent vehicle }\end{array}$ & Average to implement in a mobile intelligent vehicle \\
\hline
\end{tabular}

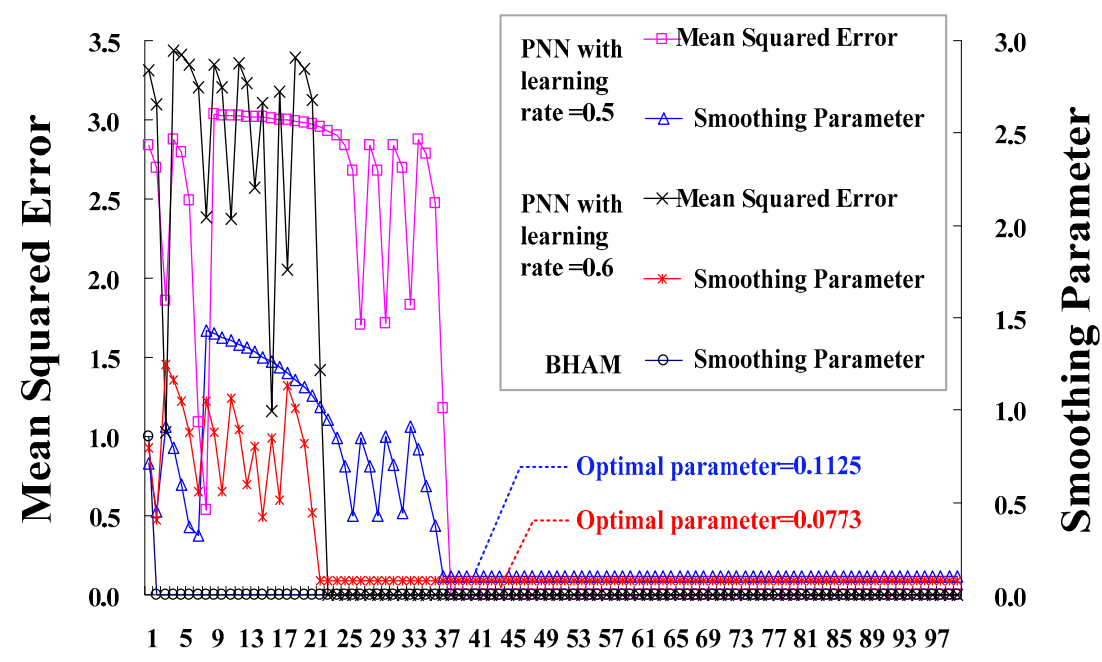

Iteration Number

Figure 8. Smoothing parameter and mean squared error versus the iteration number for PNN (learning rate $=0.5$ or 0.6$)$ and BHAM methods. 


\section{Conclusions}

In this study, we propose a multiple fault location method for use in PV array inspection employing BHAM networks. For a rooftop PV system with two strings in parallel, experimental results show that the proposed method has computational efficiency and high accuracy when conducting online electrical examinations. The power degradations were parameterized using the $\mathrm{Z}$ sigmoidal and S sigmoidal functions to screen the operation state of each PV panel. Then, BHAM method deals with the state vector to locate the faulted PV panel in a PV array. In contrast with the PNN method and traditional BHAM, the BHAM network requires minor iterative computations $(\leq 2)$ without updating network parameters, and needs no rule-based weighted inference. The modified BHAM method overcomes the nonlinear separable problem, and designs a nonlinear recurrent hetero-associative memory model. It took approximately $<0.03 \mathrm{~s}$ to complete the recall stage for a real-time application. It is thought that this method can also overcome the complexity involved in an adjustable pattern mechanism; its model can store high-dimensional training patterns in a connecting and associate matrix as modeling human cognitive and memory processes. The matrix operations require only a short design cycle, and it is easily implemented in a portable embedded system or a mobile intelligent vehicle. The proposed detect method was promising for locating faults in a PV array when dealing with solar radiation levels of $0.2 \mathrm{~kW} / \mathrm{m}^{2}-0.8 \mathrm{~kW} / \mathrm{m}^{2}$ and temperatures of $25^{\circ} \mathrm{C}-40{ }^{\circ} \mathrm{C}$, and indicated a fault location accuracy of $100 \%$ under clear days, lower solar radiation conditions, and night-to-day transition. In addition, as the capacity of PV systems will increase when connecting several PV panels in either parallel or series, and this will use a vast amount of space and long-term outdoor operations, the promising technology presented here can be integrated into light, unmanned vehicles and wireless communication. In addition, through an advanced metering infrastructure (AMI), metering power data can be transited to the BHAM-based monitor via WiFi wireless. In each PV array (parallel or series configuration), multiple BHAM-based monitoring system can be further established to identify fault types for each PV panel, including lower/upper grounded faults, open-circuit faults, bridged faults, and mismatch faults. The proposed BHAM algorithm is easy to implement and embed in existing supervisory control and data acquisition (SCADA) systems and AMI system without the need for extra devices.

Author Contributions: The model analysis on the multiple fault location in a photovoltaic array was made by L.-Y.C.; N.-S.P. and M.-H.C. The model algorithm was designed by C.-H.L.; J.-L.C. and C.-L.K. and also carried out the simulations and experiments. L.-Y.C. was responsible for writing the paper and serves as the corresponding author.

Funding: This research is funded in part by the Ministry of Science and Technology, Taiwan, under contract number: MOST 105-2634-F-244-001, duration: 1 November 2016 31 October 2017.

Conflicts of Interest: The authors declare no conflict of interest

\section{References}

1. Lin, C.H.; Huang, C.H.; Du, Y.C.; Chen, J.L. Maximum photovoltaic power tracking for the PV array using the fractional-order incremental conductance method. Appl. Energy 2011, 88, 4840-4847. [CrossRef]

2. Chiu, C.-S.; Ouyang, Y.-L. Robust maximum power racking control of uncertain photovoltaic systems: A unified T-S Fuzzy model-based approach. IEEE Trans. Control Syst. Technol. 2011, 19, 1516-1526. [CrossRef]

3. Kuo, C.-L.; Lin, C.-H.; Yau, H.-T.; Chen, J.-L. Using self-synchronization error dynamics formulation based controller for maximum photovoltaic power tracking in micro-grid systems IEEE J. Emerg. Sel. Top. Circuits Syst. 2013, 3, 459-467. [CrossRef]

4. Chao, K.-H.; Liao, B.-J.; Hung, C.-P. Applying a cerebellar model articulation controller neural network to a photovoltaic power generation system fault diagnosis. Int. J. Photoenergy 2013, 2013, 839621. [CrossRef]

5. Zhao, Y. Fault Analysis in Solar Photovoltaic Arrays. Master's Thesis, Northeastern University, Boston, MA, USA, 2010. 
6. Kuo, C.-L.; Chen, J.-L.; Chen, S.-J.; Kao, C.-C.; Yau, H.-T.; Lin, C.-H. Photovoltaic energy conversion system fault detection using fractional-order color relation classifier in micro-distribution systems. IEEE Trans. Smart Grid 2016. in Press. [CrossRef]

7. Zhao, Y.; Lehman, B.; DePalma, J.F.; Mosesian, J.; Lyons, R. Fault evolution in photovoltaic array during night-to-day transition. In Proceedings of the 2010 IEEE 12th Workshop on control and Modeling for Power Electronics, Boulder, CO, USA, 28-30 June 2010; pp. 1-6.

8. Testo, Inc. Practical Guide: Solar Panel Thermography. Available online: www.testo.com (accessed on 10 March 2018).

9. Kaplani, E. Detection of degradation effects in field-aged c-Si solar cells through IR thermography and digital image processing. Int. J. Photoenergy 2012, 2012, 396792. [CrossRef]

10. Kumar, S.; Sarkar, B.; Nija, K.S. Quality improvement of PV modules by electroluminescence and thermal Imaging. Int. J. Eng. Adv. Technol. 2014, 3, 422-427.

11. Chen, J.-L.; Kuo, C.-L.; Chen, S.-J.; Kao, C.-C.; Zhan, T.-S.; Lin, C.-H.; Chen, Y.-S. DC-side fault detection for photovoltaic energy conversion system using fractional-order dynamic error based fuzzy Petri net integrating with intelligent meters. IET Renew. Power Gener. 2016, 10, 1318-1327. [CrossRef]

12. Davarifar, M.; Rabhi, A.; El Hajjaji, A. Comprehensive modulation and classification of faults and analysis their effect in DC side of photovoltaic system. Energy Power Eng. 2013, 2013, 230-236. [CrossRef]

13. Li, H.; Liu, Z.; Liu, K.; Zhang, Z. Predictive power of machine learning for optimizing solar water heater performance: The potential application of high-throughput screening. Int. J. Photoenergy 2017, 2017, 4194251. [CrossRef]

14. Li, H.; Liu, Z. Performance prediction and optimization of solar water heater via a knowledge-based machine learning method. In Handbook of Research on Power and Energy System Optimization; IGI Global: Hershey, PA, USA, 2018; pp. 55-74.

15. Chartier, S.; Boukadoum, M. A bidirectional heteroassociative memory for binary and grey-level patterns. IEEE Trans. Neural Netw. 2006, 17, 385-396. [CrossRef] [PubMed]

16. Zhang, Z.; Pinto, J.; Plahl, C. Channel mapping using bidirectional long short-term memory for dereverberation in hands-free voice controlled devices. IEEE Trans. Consum. Electron. 2014, 60, 525-533. [CrossRef]

17. Berberian, N.; Aamir, Z.; Helie, S. Encoding sparse features in a bidirectional associative memory. In Proceedings of the 2016 International Joint Conference on Neural networks, Vancouver, BC, Canada, 24-29 July 2016.

18. Tao, D.; Wen, Y.; Hong, R. Multicolumn bidirectional long short-term memory for mobile devices-based human activity recognition. IEEE Internet Things J. 2016, 3, 1124-1134. [CrossRef]

19. Pandey, A.; Dasgupta, N.; Mukkerjee, A.K. High-performance algorithms for drift avoidance and fast tracking in solar MPPT system. IEEE Trans. Energy Convers. 2008, 23, 681-689. [CrossRef]

20. Otieno, C.A.; Nyakoe, G.N.; Wekesa, C.W. A neural Fuzzy based maximum power point tracker for a photovoltaic system. In Proceedings of the 2009 IEEE AFRICON, Nairobi, Kenya, 23-25 September 2009; pp. 1-6.

21. Buerhop, C.; Pickel, T.; Dalsass, M. Air-PV-check: A quality inspection of PV-power plants without operation interruption. In Proceedings of the 2016 IEEE 43rd Photovoltaic Specialists Conference, Portland, OR, USA, 5-10 June 2016.

22. Aghaei, M.; Dolara, A.; Leva, S. Image resolution and defects detection in PV inspection by unmanned technologies. In Proceedings of the 2016 Power and Energy Society General Meeting, Boston, MA, USA, 17-21 July 2016.

23. Quater, P.B.; Grimaccia, F.; Leva, S. Light unmanned aerial vehicles (UAVs) for cooperative inspection of PV plants. IEEE J. Photovolt. 2014, 4, 1107-1113. [CrossRef]

24. Specht, D.F. A general regression neural network. IEEE Trans. Neural Netw. 1991, 2, 568-576. [CrossRef] [PubMed]

25. Wu, J.X.; Lin, C.H.; Du, Y.C.; Chen, T. Sprott chaos synchronization classifier for diabetic foot peripheral vascular occlusive disease estimation. IET Sci. Meas. Technol. 2012, 6, 533-540. [CrossRef]

26. Huang, C.-H.; Lin, C.-H.; Kuo, C.-L. Chaos-synchronization based detector for power quality disturbances classification in a power system. IEEE Trans. Power Deliv. 2011, 26, 944-953. [CrossRef] 
27. Li, H.; Zhang, Z.; Liu, Z. Application of artificial neural networks for catalysis: A review. Catalysts 2017, 7, 306. [CrossRef]

28. Institute of Electrical and Electronics Engineers. IEEE Std. 802.11-2007: Wireless LAN Medium Access Control (MAC) and Physical Layer (PHY) Specifications; Institute of Electrical and Electronics Engineers: Piscataway, NJ, USA, 2007.

(C) 2018 by the authors. Licensee MDPI, Basel, Switzerland. This article is an open access article distributed under the terms and conditions of the Creative Commons Attribution (CC BY) license (http:/ / creativecommons.org/licenses/by/4.0/). 